\title{
Concevoir des ressources pour enseigner, un levier pour le développement des compétences informationnelles des professeurs?
}

\section{Designing resources for teaching, a lever for the development of teachers' information literacy skills?}

\author{
Anita Messaoui ${ }^{1}$ a \\ ${ }^{1}$ S2HEP / ACTé - ÉducTice, Institut français de l'éducation (IFÉ) \\ ENS de Lyon, 15 parvis René Descartes, BP 7000, 69342 Lyon Cedex 07 - France
}

\begin{abstract}
Résumé. Les enseignants français conçoivent depuis longtemps leurs ressources d'enseignement. Avec l'accroissement des ressources numériques, ces tâches de conception se sont complexifiées. Quelles sont les compétences informationnelles en jeu dans le travail de conception des ressources d'enseignement? Peut-on définir une expertise documentaire des enseignants en rapport avec leur travail sur les ressources? Notre contribution propose de répondre à ses questions en nous basant sur l'analyse du travail documentaire d'une enseignante d'anglais de collège pendant la préparation d'une nouvelle leçon. À partir de l'approche documentaire du didactique et du concept de schème, deux schèmes documentaires ont été identifiés et caractérisés : «sélectionner une ressource» et «garder la trace d'une ressource». Des connaissances et des règles d'action associées à ces schèmes ont été mises en évidence, prouvant que ces deux schèmes constituent une mise en action des compétences « évaluer une information » et « retrouver une information».
\end{abstract}

\begin{abstract}
In France, teachers used to design their resources from a long time. With the increase of digital resources, this design task became more complicated. What are the information literacy's skills into play in this design? How could we define a "documentational expertise" in relation to teachers' documentational work? The contribution aims to answer these questions. For this purpose, our analysis is based on the documentational work of an English teacher, in a French secondary school, preparing a new lesson. With the concept of scheme and the documentational approach to didactics, two schemes were identified and characterized, through the teacher's activities: selecting and saving a resource. Associated knowledge and rules of action for these schemes are highlighted. These two schemes constitute a putting into action of information literacy's skills: evaluate information and retrieve information.
\end{abstract}

\footnotetext{
${ }^{a}$ Auteur de correspondance : anita.messaoui@ens-lyon.fr
} 


\section{Introduction}

L'éducation aux médias et à l'information (EMI) est apparue en 2013 dans les textes officiels français. Fin 2015, le ministère de l'Éducation nationale a publié un référentiel de compétences pour l'EMI avec les programmes qui accompagnent la réforme $2016 \mathrm{du}$ collège. Cette «éducation à » est un enseignement transversal intégré au socle commun de connaissances, de compétences et de culture ${ }^{1}$. Elle est fortement sollicitée dans les dispositifs associés à la réforme, comme les Enseignements Pratiques Interdisciplinaires (EPI) et l'Éducation Morale et Civique (EMC). Tous les professeurs sont en charge de la dispenser, avec l'appui du professeur documentaliste. Cependant, les enseignants disciplinaires ne sont pas des spécialistes du domaine. Ils n'ont, le plus souvent, aucune formation dans le champ des médias et de l'information. Warzager (2003) a mené une étude d'envergure dans les IUFM ${ }^{2}$ (Institut Universitaire de Formation des Maîtres) qui montre que $87 \%$ des professeurs stagiaires du secondaire n'ont pas reçu de formation documentaire ${ }^{3}$. Or, les enseignants ont tous des pratiques informationnelles en lien avec leur activité professionnelle (Liquète, 2001; Messaoui, 2016), en particulier lors des activités de préparation des cours. Les ressources sont la matière première du professeur. Il les recherche, les rassemble, les transforme, les nourrit de ses préoccupations didactiques, de sa connaissance des élèves, pour concevoir, au terme de ce travail documentaire, un document qui constitue la matière du travail avec les élèves. Par conséquent, les enseignants ne sont pas dépourvus de compétences informationnelles. Leur expertise, essentiellement basée sur leurs pratiques et leurs expériences, est souvent implicite et relève d'un apprentissage empirique.

L'objet de notre contribution, qui s'inscrit dans l'axe 1 du colloque faire avec et faire sans, les ressources mobilisées, est de comprendre comment les enseignants développent leurs compétences informationnelles par leur travail documentaire et leurs interactions avec les ressources, en particulier pendant les activités de conception. La problématique traitée dans cette contribution explore deux axes complémentaires : quelles sont les compétences informationnelles qui interviennent dans la conception d'une leçon et comment les compétences informationnelles contribuent-elles au développement de l'expertise documentaire. Le concept d'expertise documentaire est émergent. Nous en proposons une définition à la fin de notre exposé.

La première section présente les concepts et les approches théoriques qui sous-tendent notre analyse. À partir de ce contexte théorique, nous avons mis en place une méthodologie qui s'appuie sur les principes de l'investigation réflexive (Gueudet et Trouche, 2010) et défini notre terrain de recherche pour réaliser une étude de cas. Ensuite, nous présentons les premiers schèmes que nous avons identifiés dans une situation de conception d'une nouvelle leçon : sélectionner une ressource et garder la trace d'une ressource.

\footnotetext{
${ }^{1}$ http://www.education.gouv.fr/pid25535/bulletin_officiel.html?cid_bo=87834

${ }^{2}$ Ces structures de formation des enseignants sont remplacées par les ESPE depuis 2013

${ }^{3}$ La situation a peut-être évoluée en France avec la mise en place du C2i enseignant, mais aucune étude récente ne s'intéresse à la question de la formation documentaire
} 


\section{Contexte théorique}

L'analyse des interactions entre les enseignants et les ressources prend appui sur des concepts issus des sciences de l'éducation et de sciences de l'information et de la communication (SIC). Nous présentons d'abord l'approche documentaire du didactique (écrit ADD par la suite) (Gueudet et Trouche, 2010), puis les apports de la didactique professionnelle avec la théorie de la conceptualisation dans l'action et le concept de schème (Pastré, Mayen et Vergnaud, 2006; Vergnaud, 2011) et enfin les compétences informationnelles et l'information literacy qui relève du champ des SIC.

\subsection{L'approche documentaire du didactique}

Le terme de ressource a été beaucoup sollicité au cours de ce colloque et les définitions proposées sont nombreuses. Nous commençons donc par définir ce que nous entendons par « ressource» dans notre contexte théorique. Les travaux d'Adler (2000), repris par Gueudet et Trouche (2010), lui donne une acceptation relativement large : tout ce qui permet de resourcer l'activité de l'enseignant. Pour notre part, afin d'observer le développement des compétences informationnelles, nous restreignons cette définition à toutes les ressources matérielles susceptibles de soutenir l'enseignant dans son activité professionnelle, mais nous conservons du verbe «re-sourcer» l'accent mis sur l'usage en contexte de la ressource comme moyen pour l'enseignant de transformer son activité. Les ressources mobilisées par les enseignants sont structurées, en fonction de leurs activités, dans un système de ressources.

Le travail documentaire des enseignants est également un des concepts fondateurs de l'ADD. Gueudet et Trouche (2010) le définissent comme un processus articulant étroitement la conception et la mise en œuvre des ressources d'enseignement par les professeurs. Le travail documentaire englobe en particulier la recherche de ressources, leur appropriation, et leur intégration dans un système de ressources. En interagissant avec les ressources, les enseignants les transforment et se transforment dans un double processus d'instrumentation et d'instrumentalisation. Au terme de ce processus, nommé genèse documentaire, l'enseignant a conçu un document, une entité hybride composée de ressources combinées et de schèmes, c'est-à-dire d'usages potentiels, d'objectif d'enseignement et des connaissances qui sous-tendent ces usages. Le concept de schème est aussi central dans la théorie de la conceptualisation dans l'action (voir §2.2).

Nous émettons l'hypothèse que le développement professionnel, intimement lié à la genèse documentaire (Gueudet et Trouche, 2010), est favorisé lorsque le sujet se trouve dans une situation nouvelle. Les derniers développements de l'ADD (Pepin et al., 2017) accordent une place importante à la capacité de conception des enseignants et à ses composantes : "teacher design can be described as the creation of "something new" (e.g. combining existing and novel elements) as a deliberate/conscious act in order to reach a certain (didactical) aim.» (p. 4). En conséquence, l'activité de préparation d'une nouvelle leçon est une situation permettant d'observer la conception de nouvelles ressources et potentiellement, le développement de nouvelle connaissance.

\subsection{Conceptualisation dans l'action et schème}

Dans leurs travaux de développement d'une didactique professionnelle, Pastré, Mayen et Vergnaud (2006) soutiennent que les connaissances naissent dans l'action. Selon eux, il existe des savoirs basés sur l'expérience, appelés connaissances opératoires, et des savoirs basés sur l'apprentissage, nommés connaissances prédicatives. Les premiers sont dans le registre du faire, les seconds dans celui de l'explicitation. Notre postulat de départ est le 
suivant : les compétences informationnelles appartiennent à la catégorie des savoirs qui se développent dans l'action.

Tout comme l'ADD, la conceptualisation dans l'action s'appuie sur l'analyse de l'activité, pour identifier les compétences mises en œuvre par les acteurs. Or, si on ne se contente pas de considérer le résultat de l'activité, c'est-à-dire la performance, mais l'activité elle-même ainsi que sa forme, la notion de compétence n'est pas suffisante. Les chercheurs en didactique professionnelle lui préfèrent le concept de schème, qui est alors plus opérationnel pour décrire et analyser les formes d'organisation de l'activité en situation. C'est ce que nous faisons pour le travail documentaire.

Vergnaud (2011) a repris de Piaget le concept de schème, qu'il définit comme une totalité dynamique fonctionnelle, en le couplant au concept de classe de situations : "le schème est une organisation invariante de la conduite pour une classe donnée de situations » (p. 283). Le schème pilote l'activité pour réaliser un type de tâches, pour une classe de situations donnée. Vergnaud caractérise le schème selon quatre composants qui interagissent entre eux pour mener à bien l'activité. Le premier composant du schème est intentionnel : un but clairement identifié, des intentions et des sous-buts. Ensuite, la partie générative du schème est constituée des règles d'action, de prise d'information et de contrôle. Troisième composant, les invariants opératoires sont un élément déterminant pour que le schème puisse effectivement conduire l'activité ; ce terme désigne les connaissancesen-acte, c'est-à-dire les propositions tenues pour vraies ou pertinentes dans une classe de situations. Enfin les situations d'une même classe étant proches, mais jamais identiques, le schème se compose également des inférences qui permettent au sujet de s'adapter. Les inférences jouent un rôle majeur dans la dynamique de développement des schèmes.

Nous souhaitons introduire ici une distinction opérée par Rabardel (1995) entre le schème d'action instrumenté, entité globale, qui permet de réaliser le but principal de l'activité et le schème d'usage, qui conduit les tâches secondaires indispensables à la réalisation du but principal. Par ailleurs, un schème d'usage peut être mobilisé dans différentes classes de situations. Bien que les schèmes documentaires soient impliqués dans toutes les activités du travail documentaires, nous restreignons notre étude à la caractérisation de schèmes d'usages impliqués dans la mise en œuvre des compétences informationnelles.

\subsection{Information literacy et compétences informationnelles}

Nous ne rentrons pas dans les débats de traduction de l'expression information literacy, ni sur 1'historique du terme (Boustany, 2014; Karsenti et al., 2014), nous utilisons dans l'étude le terme de compétences informationnelles en nous appuyant sur la vision portée par l'Association of College and Research Libraries (ACRL) et l'Information Literacy Competency Standards for Higher Education (ACRL, 2000). Mondialement reconnu, ce référentiel de compétences a servi de socle pour la construction de nombreuses formations de l'enseignement supérieur (ADBU, 2012; CREPUQ, 2005). Il définit quatre domaines compétences. Le premier est la capacité à identifier et définir son besoin d'information, c'est-à-dire être capable de cerner précisément son sujet et l'information recherchée. Le second domaine recouvre la démarche de recherche: trouver l'information, savoir la rechercher et la localiser. Le troisième domaine englobe les stratégies d'évaluation et de sélection de l'information et le dernier la capacité à utiliser l'information pour répondre aux besoins initialement identifiés. Ce référentiel, très imprégné du fonctionnement des bibliothèques, propose une vision procédurale des compétences informationnelles.

L'essor d'Internet et surtout des médias sociaux depuis quelques années ont fait évoluer les pratiques informationnelles des étudiants et des chercheurs, cela a conduit cette association à actualiser son référentiel. En 2015, l'ACRL a publié un nouveau cadre pour 
l'enseignement de l'information literacy à l'université (ACRL, 2015). Beaucoup moins orienté vers les procédures, ce cadre pose davantage l'information literacy comme une posture intellectuelle : l'autorité, qui est un des critères de l'évaluation, y est envisagée comme une construction, la création du savoir comme un échange, la recherche comme une enquête, la production d'information comme un processus. Or, toutes ces attitudes sont mobilisées dans le travail documentaire.

Malgré la refonte du référentiel de compétences proposée par l'ACRL, l'aspect de la gestion de ses informations n'est pas considéré dans le référentiel de 2015. Pour trouver une description, il faut nous tourner vers le champ de la gestion personnelle de l'information. Jones (2007) définit 3 grands domaines : (1) trouver / retrouver l'information pour répondre à un besoin identifié, selon une conception proche du modèle proposer par l'ACRL, (2) garder l'information au sein de son système personnel d'information (SPI) et (3) gérer et organiser ses collections au sein de son SPI. L'approche de Jones introduit une dimension très présente dans le travail documentaire qui est la conservation et l'organisation des ressources produites et collectées par les enseignants.

Pour conclure, le champ des compétences informationnelles recouvre un large panel d'activités qui inclut aussi bien le processus de la recherche d'information que l'organisation des ressources au sein d'un système structuré. Suivant la proposition de Vergnaud qui substitue le concept de schème à la notion de compétences, notre contribution propose d'identifier des schèmes documentaires associés aux compétences informationnelles dans la situation de préparation d'une nouvelle leçon.

\section{Une méthodologie basée sur des études de cas}

Cette section présente les principes de l'investigation réflexive avant de décrire les caractéristiques de notre terrain et se conclut sur les méthodes d'analyse mise en œuvre.

La méthodologie élaborée ici prend appui sur l'investigation réflexive (Gueudet et Trouche, 2010) dont les principales caractéristiques sont : un suivi sur le long terme afin de mesurer les éléments de stabilité et de variation dans l'activité ; un suivi dans et hors la classe pour saisir tous les moments du travail documentaire; une collecte de données étendue, depuis les ressources produites et utilisées par l'enseignant aux vidéos de l'activité de l'enseignant; une implication de l'enseignant dans le suivi et la collecte des données qui l'amène à expliciter son activité et développer une posture réflexive sur son travail. Dans le respect des quatre principes précédemment évoqués, nous avons construit une méthodologie originale qui se déploie au cours de deux années de suivis.

Afin de prendre connaissance de l'environnement dans lequel évolue l'enseignant, le premier entretien consiste en une visite guidée des ressources. Il s'agit d'un entretien semidirectif, au domicile de l'enseignant, où le chercheur utilise une instruction au sosie pour lui demander de décrire ses ressources, leur organisation et leur usage. Introduite en psychologie du travail par Clot (2001) et reprise par Gueudet et Trouche (2010), l'instruction demande à l'enseignant d'expliquer à une personne qui va le remplacer comment sont organisées ses ressources et comment il les utilise. L'instruction au sosie est " moyen détourné, un contact social artificiel avec soi-même. Il autorise une ré-entrée dans l'action, une répétition sans répétition, la mise au travail de l'action dans une autre activité avec le sosie où elle sert maintenant de ressource. La conscience est ce dédoublement du vécu, revécu pour vivre autre chose » (Clot, $2001: 261$ ).

Nous proposons ensuite de capter l'activité de l'enseignant à trois moments du travail documentaire : lors de la conception d'une nouvelle leçon, pendant sa mise en œuvre en classe et pendant le processus de révision de la ressource. Dans le prolongement de l'investigation réflexive, nous demandons à l'enseignant de se filmer, seul, à son domicile pendant la préparation d'une leçon dont le sujet a été choisi en concertation avec le 
chercheur. Dans cette phase, l'enseignant devient producteur des données de la recherche. Le dispositif mis en place permet d'enregistrer à la fois ce qui se passe sur l'écran de son ordinateur et son visage avec la webcam ; l'enseignant explique ce qu'il fait en même temps qu'il prépare sa leçon. Ce dispositif vise à renforcer sa posture réflexive. Dans un deuxième moment, le chercheur vient observer la mise en œuvre de la leçon en classe, et réalise un enregistrement vidéo. Dans un troisième moment, un an plus tard, l'enseignant se filme pendant la révision de la leçon.

Notre terrain d'étude s'est constitué autour d'un collège en milieu rural. Nous avons mis en place le suivi longitudinal décrit ci-dessus pour trois enseignantes : deux en anglais et une en mathématique. L'étude de cas exposée concerne la situation de préparation d'une leçon d'une professeure d'anglais, Ludivine, leçon qui porte sur le présent BE+ING pour sa classe de $6^{\mathrm{e}}$ (figure 1 ).

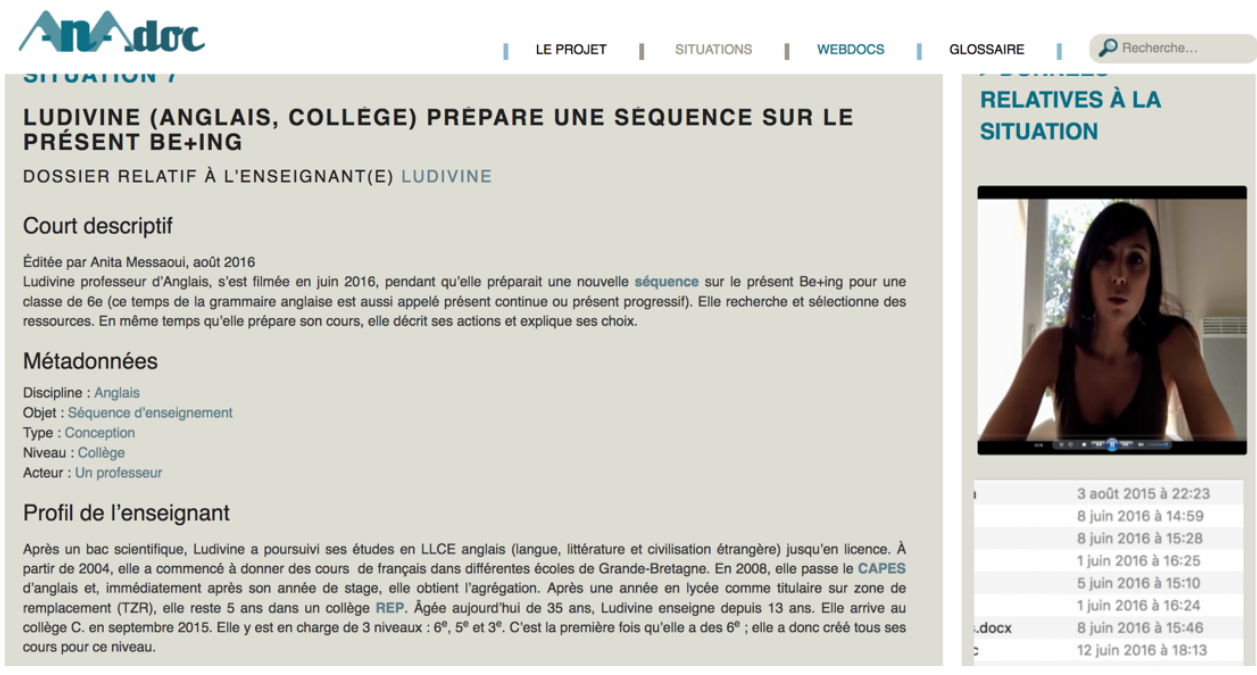

Figure 1. Présentation de la situation de Ludivine sur AnA.doc.

Elle s'est filmée deux fois. Une première fois lors de la création de la séquence et un an plus tard au moment de la révision. En plus des vidéos, Ludivine nous a aussi transmis le dossier contenant toutes les ressources associées à cette séquence. C'était la première fois que cette enseignante, nouvellement arrivée dans l'établissement, était responsable d'une classe de $6^{\mathrm{e}}$ La première année, elle a recherché des ressources, d'abord dans plusieurs manuels scolaires, puis sur YouTube pour obtenir des ressources authentiques. La situation correspond donc à la préparation d'une nouvelle leçon. Suivant la définition donnée plus haut (\$2.1), il s’agit d'une activité de conception de ressources pour enseigner. Lors de la révision de la séquence, l'enseignante a surtout vérifié la pertinence des ressources sélectionnées l'année précédente et a cherché des ressources complémentaires pour s'adapter à la nouvelle situation de classe. À partir des données collectées lors de ces situations, nous avons caractérisé des schèmes spécifiques aux compétences informationnelles.

Les données vidéo collectées (discours et actions) sont analysées et codées avec le logiciel Atlas.ti. La grille de codage (tableau 1) croise les compétences informationnelles identifiées, le type de ressource mobilisée (site Internet, manuel, logiciel...) et les différents composants du schème. Elles font ensuite l'objet d'une publication sur la plateforme 
AnA.doc ${ }^{4}$ (voir la contribution de Rocha à ce colloque). Il s'agit d'une plateforme web, hébergée par l'ENS de Lyon, qui permet de stocker les données de la recherche, publier des analyses et partager l'ensemble avec la communauté de recherche impliquée dans le projet ReVEA $^{5}$ (Alturkmani et al., à paraître). AnA.doc est structuré en 3 niveaux. Le niveau situation présente l'ensemble des données associées à une situation de collecte ainsi que la description de la méthodologie et des questions de recherche. Le niveau webdocument présente des analyses de la situation en s'appuyant sur de courts extraits. Le dernier niveau est le glossaire qui propose les définitions des concepts théoriques mobilisés dans les analyses.

Tableau 1. Grille de codage utilisée dans l'analyse des vidéos de Ludivine

\begin{tabular}{|l|c|c|}
\hline \multicolumn{1}{|c|}{ Code } & $\begin{array}{c}\text { Vidéo 1 : } \\
\text { préparation }\end{array}$ & $\begin{array}{c}\text { Vidéo 2 : } \\
\text { révision }\end{array}$ \\
\hline Analyser / Évaluer & 5 & 5 \\
\hline Buts/intention & 3 & 1 \\
\hline Classer / organiser & 2 & 2 \\
\hline Créer une ressource & 0 & 1 \\
\hline Enregistrer/ sauvegarder / prendre en note & 6 & 4 \\
\hline Identifier ses besoins & 3 & 2 \\
\hline Manuels scolaires (papier ou numérique) & 2 & 1 \\
\hline Mise en forme de l'information & 0 & 1 \\
\hline Rechercher / retrouver & 4 & 6 \\
\hline Recyclage de ses ressources propres & 1 & 5 \\
\hline Sélectionner & 5 & 5 \\
\hline Site Internet & 2 & 4 \\
\hline
\end{tabular}

\section{Essai de caractérisation de schèmes documentaires}

En nous basant sur les situations de préparation d'une leçon de Ludivine et la régularité des actions observées, nous proposons de caractériser deux schèmes documentaires impliqués dans le processus de conception d'une nouvelle ressource : «sélectionner une ressource » et "garder la trace d'une ressource». Pour chacun nous avons recherché les quatre composants définis par Vergnaud (voir §2.2).

\subsection{Le schème « sélectionner une ressource »}

Le but de l'activité "préparer une leçon» est de choisir les ressources qui serviront de support à l'apprentissage des élèves. Pour cela, le professeur va évaluer différentes ressources afin de déterminer s'il les sélectionne ou non pour concevoir sa leçon. C'est pourquoi nous affirmons qu'un des sous-buts de cette activité est de déterminer la valeur d'une information.

Tout au long des films d'étude, le professeur explique pourquoi elle retient telle ressource et rejette telle autre, ce faisant, elle explicite ses critères de choix. En relevant ces éléments dans son discours, nous avons déterminé plusieurs catégories de connaissances

\footnotetext{
${ }^{4}$ Pour des raisons de confidentialité, l'accès à la plateforme nécessite une authentification par des codes. Contactez l'auteure de l'article pour les obtenir.

${ }^{5} \mathrm{https}$ ///www.anr-revea.fr/
} 
mobilisées par Ludivine pour argumenter ses choix (tableau 2). Il y a tout d'abord les connaissances liées à la discipline enseignée, ici des connaissances sur la langue anglaise (grammaire, vocabulaire, prononciation) pour évaluer la qualité linguistique de la ressource ; ensuite des connaissances d'ordre didactique autour des contenus à enseigner et de leur progression; les éléments relevant des programmes scolaires (connaissance curriculaire) sont aussi invoqués pour justifier la sélection; la connaissance des élèves (niveau, difficulté) est également une dimension importante; enfin, dans la dernière colonne, regroupe les connaissances sur le contexte d'enseignement de Ludivine.

En ayant accès à la vidéo de l'activité, c'est-à-dire en pouvant observer le déroulement de l'action, nous avons également pu déterminer des règles d'action. Tout au long des vidéos de préparation et de révision de la leçon, certaines actions se répètent dans un ordre identique. Nous en inférons des règles d'action, de prise d'information et de contrôle, qui correspondent à l'analyse de la ressource, la décision de sélectionner ou non la ressource étant entièrement dépendante de cette analyse.

Tableau 2. Récapitulatif des connaissances mobilisées par l'enseignante pour la tâche de sélection

\begin{tabular}{|l|l|l|l|l|}
\hline $\begin{array}{c}\text { Connaissances } \\
\text { disciplinaires }\end{array}$ & $\begin{array}{l}\text { Connaissances } \\
\text { didactiques }\end{array}$ & $\begin{array}{l}\text { Prescriptions } \\
\text { des } \\
\text { programmes }\end{array}$ & $\begin{array}{l}\text { Connaissances } \\
\text { des élèves }\end{array}$ & $\begin{array}{l}\text { Connaissances } \\
\text { du contexte }\end{array}$ \\
\hline Grammaire & $\begin{array}{l}\text { Progression de } \\
\text { l'enseignement } \\
\text { sur l'année en } \\
\text { cours et les } \\
\text { années } \\
\text { suivantes. } \\
\text { Vocabulaison }\end{array}$ & $\begin{array}{l}\text { Utiliser une } \\
\text { variété de } \\
\text { support : texte, } \\
\text { vidéo, son. } \\
\text { Utiliser des } \\
\text { ressources } \\
\text { authentiques. } \\
\text { Prononciation } \\
\text { exercices par } \\
\text { rapport aux } \\
\text { buts. }\end{array}$ & $\begin{array}{l}\text { Capacité } \\
\text { d'attention } \\
\text { tâche finale } \\
\text { Difficultés } \\
\text { généralement } \\
\text { rencontrées }\end{array}$ & $\begin{array}{l}\text { Présence } \\
\text { d'enceinte et } \\
\text { d'un } \\
\text { vidéoprojecteur } \\
\text { dans la salle de } \\
\text { classe }\end{array}$ \\
\hline
\end{tabular}

La règle d'action identifiée est la suivante : l'enseignante commence par prendre connaissance du contenu, que ce soit du texte, une piste audio ou une vidéo. Elle compare ensuite ce contenu avec les objectifs d'enseignement qu'elle s'est fixés ou les besoins d'information qu'elle a auparavant identifiés. Après quoi, elle évalue la ressource à l'aune des catégories de connaissances que nous avons mises en évidence dans le tableau 2. Enfin, le professeur décide de sélectionner ou non cette ressource, ou encore de la garder de manière provisoire en attendant une nouvelle analyse. Au travers de cette succession d'action, il s'agit de vérifier la pertinence du contenu des ressources analysées en regard des attentes de l'enseignante. Les connaissances exprimées par l'enseignante guident son action, nous pouvons alors les considérer comme de potentiels invariants opératoires.

Le schème sélectionner une ressource met en jeu un processus d'évaluation des informations qui correspond à une des compétences informationnelles définies par le référentiel ACRL : évaluer l'information.

\subsection{Le schème " garder la trace d'une ressource »}

Le second schème que nous avons identifié et caractérisé pour ces situations de préparation d'une leçon est garder la trace d'une ressource. Cette action est la première étape de l'intégration d'une nouvelle ressource dans le système de ressource de l'enseignant. Lors du processus de conception d'une nouvelle leçon, l'enseignante sélectionne des ressources et 
les conserve afin de les combiner à d'autres pour créer son document. Un des buts de ce schème est de mettre une ressource sélectionnée en sécurité afin de la retrouver rapidement lorsque le professeur en aura besoin.

Pour atteindre ce but, l'enseignante met en œuvre différentes stratégies qui peuvent être considérées comme autant d'inférence du schème garder la trace d'une ressource: elle prend en note sur une feuille des références d'exercices de manuels scolaires, elle enregistre des fichiers sur son ordinateur, elle enregistre aussi des liens vers des pages Internet. L'identification des caractéristiques de ce schème, se base surtout sur la gestuelle du professeur, accessible grâce à la vidéo. Les actions associées à cette tâche ne sont presque jamais commentées par le professeur. Pourtant, ces actions sont bien sous-tendues par des connaissances que nous avons isolées. Nous inférons que les actions de l'enseignante reposent sur des connaissances qui ne sont pas explicitées, nous les qualifions de connaissances opératoires (Pastré et al., 2006). Nous les classons en 3 catégories (tableau 3).

Tableau 3. Tableau récapitulatif des connaissances mobilisées par l'enseignante pour la tâche de garder la trace d'une ressource.

\begin{tabular}{|l|l|l|}
\hline $\begin{array}{c}\text { Métadonnées pour } \\
\text { identifier une ressource }\end{array}$ & \multicolumn{1}{|c|}{$\begin{array}{c}\text { Propriété d'une } \\
\text { ressource digitale }\end{array}$} & $\begin{array}{c}\text { Connaissance du système } \\
\text { de classement }\end{array}$ \\
\hline Titre & Copier & $\begin{array}{l}\text { Arborescence des dossiers } \\
\text { numériques }\end{array}$ \\
Auteur & Enregistrer & Système de nommage des \\
Éditeur & Nommer/renommer & fichiers \\
Pagination & Créer et déplacer des & \\
Adresse URL & fichiers & \multicolumn{2}{|l}{} \\
\hline
\end{tabular}

Tout d'abord, l'action du professeur est sous-tendue par la connaissance des métadonnées qui servent à identifier une ressource, l'enseignante note sur une feuille les titres des manuels dans lesquelles elle prélève des exercices, Round the corner ${ }^{6}$ et Enjoy English. Elle écrit également les numéros et les pages auxquelles se trouvent les exercices qu'elle a sélectionnés. D'autres connaissances sont mobilisées comme l'adresse URL d'une page web ou encore la notion d'auteur pour retrouver une vidéo sur YouTube. Par ailleurs, l'usage des ressources numériques mobilise aussi des connaissances en rapport avec les propriétés intrinsèques à une ressource numérique : savoir copier-coller une image ou un texte, renommer un fichier, créer et déplacer un dossier ou un fichier. Toutes ces connaissances non explicitées sont nécessaires à la conduite de l'activité. La dernière catégorie concerne la connaissance de Ludivine au sujet de son propre système de classement (arborescence et système de nommage). Le professeur ne pourrait pas garder la trace des ressources sélectionnées sans toutes ces connaissances, c'est pourquoi nous suggérons de les considérer comme des invariants opératoires.

Nous avons mis en évidence des règles d'actions qui se répètent plusieurs fois pendant les situations de préparation d'une leçon, cependant, nous distinguons des procédures différentes pour les ressources numériques et les ressources papier.

Concernant les ressources numériques, l'enseignante nomme le fichier de manière à ce que son contenu soit immédiatement identifiable, par exemple «objectifs Unit 7 » ou «présent continu devoirs», puis elle enregistre chaque ressource dans un dossier qui regroupe l'ensemble des ressources nécessaires à la séquence d'enseignement. Le nom de ce dossier, Unit 7 what's going on, correspond lui-même à une règle d'action bien établie : chaque dossier de séquence est numéroté de Unit 1 à Unit 7, auquel est associé le nom du

\footnotetext{
${ }^{6}$ http://www.roundthecorner.net/round.php
} 
thème principal de la séance. La recherche d'efficacité pour retrouver les ressources est ici manifeste.

Malgré un large recours aux ressources numériques, les manuels restent pour Ludivine un matériau de base notamment pour les exercices. Ludivine repère les exercices qui correspondent à la notion travaillée et en sélectionne quelques-uns. Comme il s'agit de ressource au format papier, l'enseignante ne peut pas les intégrer directement dans ses ressources numériques. Nous observons alors une sauvegarde en deux temps. La première étape est de noter sur une feuille les références des ouvrages et des exercices afin de pouvoir les retrouver. Dans un deuxième temps, qui a été réalisé hors de l'enregistrement vidéo, le professeur a saisi les exercices dans un traitement de texte, éventuellement en les modifiant, et a enregistré ces fichiers dans le dossier «Unit 7 ».

Les règles d'action associées au schème garder la trace d'une ressource, sont à mettre en relation avec un but exprimé par l'enseignante dans l'enregistrement: accéder facilement et rapidement à ses ressources en classe. Ce schème est donc l'expression de la compétence informationnelle retrouver l'information.

\section{5 Éléments de conclusion}

Les résultats présentés dans cette étude sont une première étape de l'analyse. Les éléments des schèmes avancés, et en particulier les catégories d'invariants opératoires devront être confirmées et affinées par les analyses du travail documentaire des autres enseignants suivis.

Les premiers résultats de cette recherche montrent que des compétences informationnelles sont bien mises en œuvre dans le travail documentaire des enseignants au moment de la conception d'une nouvelle ressource. Si la notion de développement n'a pas pu être démontrée sur un suivi d'une seule année, l'acquisition des compétences informationnelles semble cependant être issue de l'expérience de l'enseignante puisqu'elle n'a pas reçu de formation spécifique. La poursuite du suivi devrait amener de nouveaux éléments.

Pour le schème «sélectionner une ressource», l'évaluation de la valeur d'une information est une aide précieuse à la décision de sélectionner ou non la ressource. Notre analyse a mis en évidence plusieurs catégories de connaissances mobilisées dans les activités de sélection d'une ressource. Nous proposons une catégorisation de ces connaissances: connaissances disciplinaires, connaissances didactiques, connaissance curriculaire, connaissance des élèves et connaissances du contexte. Ces connaissances autour de l'acte d'enseigner sont indispensables pour définir la valeur d'une information. Elles jouent un rôle déterminant dans l'expertise documentaire de cette enseignante. Si les connaissances disciplinaires et didactiques se sont constituées lors de la formation initiale, les autres catégories de connaissances sont issues de l'expérience de l'enseignante.

Pour le second schème, garder la trace d'une ressource, les règles d'actions et les connaissances mises en évidence sont en étroite relation avec les activités d'organisation du système de ressource qui permettent notamment de retrouver efficacement les ressources lorsque le besoin s'en fait sentir. Cette activité et les connaissances qui la sous-tendent relèvent de ce que Jones (2007) définit comme le niveau méta-activité de la gestion personnelle de l'information. Gérer son système d'information de manière à pouvoir retrouver efficacement ses ressources est un aspect non négligeable de l'expertise documentaire de Ludivine bien que l'enseignante n'explicite pas ces connaissances pendant l'enregistrement. Là encore, il semble que ce soit l'expérience du travail documentaire qui contribue à leur développement.

Dans les deux schèmes caractérisés dans cette contribution, nous observons une place importante des connaissances disciplinaires, didactiques et pédagogiques dans le travail 
documentaire. C'est pourquoi nous proposons de définir le concept d'expertise documentaire comme la combinaison et la mise en acte des connaissances disciplinaires, des connaissances didactiques, des connaissances curriculaires et des connaissances de l'environnement informationnelles. Ce concept est en cours de construction et cette définition devra encore être affinée, notamment en déterminant les composantes de l'expertise documentaire.

\section{Bibliographie}

Adler J. (2000). Conceptualising Resources as a Theme for Teacher Education. Journal of Mathematics Teacher Education, vol. 3, n³, p. 205-224.

Alturkmani M. D., Daubias P., Loisy C., Messaoui Anita et Trouche L. (à paraître). Instrumenter les recherches sur le travail documentaire des enseignants : le projet AnA.doc. Education \& didactique.

Association des directeurs et des personnels de direction des bibliothèques universitaires et de la documentation (ADBU) (2012). Référentiel des compétences informationnelles. URL : http://www.enssib.fr/bibliotheque-numerique/notices/60368-referentiel-descompetences-informationnelles-adbu, consulté le 25/07/2017.

Association of College \& Research Libraries (ACRL) (2000). Information Literacy Competency Standards for Higher Education (Text), American Library Association (ALA). URL : http://www.ala.org/acrl/standards/informationliteracycompetency, consulté le 25/07/2017.

Association of College and Research Libraries (ACRL) (2015). Framework for Information Literacy for Higher Education, American Library Association (ALA). URL: http://www .ala.org/acrl/sites/ala.org.acrl/files/content/issues/infolit/Framework_ILHE.pdf, consulté le 25/07/2017.

Boustany J. (2014). Le champ de l'information literacy en France à travers les publications scientifiques. In V. Liquète (éd.), Cultures de l'information, Paris : CNRS éditions, p. 139158.

Clot Y. (2001). Clinique du travail et action sur soi. In J.-M. Baudouin \& J. Friedrich, Théories de l'action et éducation. De Boeck Supérieur. URL: https://doi.org/10.3917/dbu.baudo.2001.01.0255, consulté le 25/07/2017.

Conférence des recteurs et principaux des universités du Québec (CREPUQ) (2005). Norme sur les compétences informationnelles dans l'enseignement supérieur de l'association of college \& research libraries. URL : http://www.crepuq.qc.ca/IMG/pdf/normeacrl-web-0305-v4.pdf, consulté le 25/07/2017.

Gueudet G. et Trouche L. (2010). Des ressources aux documents, travail du professeur et genèses documentaires. In G. Gueudet \& L. Trouche (Éds.), Ressources vives : Le travail documentaire des professeurs en mathématiques, Rennes/Lyon : PUR/INRP, p. 57-74.

Jones W. (2007). Personal Information Management. Annual Review of Information Science and Technology, vol. 41, $\mathrm{n}^{\circ} 1, \mathrm{p} .453-504$.

Karsenti T., Dumouchel G. et Komis V. (2014). Les compétences informationnelles des étudiants à l'heure du Web 2.0: proposition d'un modèle pour baliser les formations. Documentation et bibliothèques, vol. $60, \mathrm{n}^{\circ} 1$, p. 20-30. 
Messaoui A. (2016). Les pratiques informationnelles individuelles et collectives des enseignants d'un collège rural : une enquête exploratoire. Spirale, Suppl. électronique au $n^{\circ} 57$, p. 55-82. URL : http://www.spirale-edu-revue.fr/IMG/pdf/messaoui_anita_-_spirale_2016.pdf

Pastré P., Mayen P. et Vergnaud G. (2006). La didactique professionnelle. Revue française de pédagogie. Recherches en éducation, $\mathrm{n}^{\circ} 154, \quad \mathrm{p}$. 145-198. [https://doi.org/10.4000/rfp.157].

Pepin B., Gueudet G. et Trouche L. (2017). Refining teacher design capacity: Mathematics teachers' interactions with digital curriculum resources. ZDM-Mathematics Education, vol. 49, n 5, p. 799-812. [https://doi.org/10.1007/s11858-017-0870-8].

Vergnaud Gérard (2011). Au fond de l'action, la conceptualisation. In J. M. Barbier (éd.), Savoirs théoriques et savoirs d'action ( $3^{\mathrm{e}}$ édition), Paris : Presses Universitaires de France, p. 275-292.

Warzager Daniel (2003). Où en est la formation à l'information documentaire dans la formation des enseignants? Assises nationales pour l'éducation à l'information. Paris.

URL : http://urfist.enc.sorbonne.fr/anciensite/Assises/Ass-Warzager.rtf, consulté le 25/07/2017. 